\title{
Percolation and three-dimensional structure of supercritical water
}

\author{
M. Bernabei, ${ }^{1}$ A. Botti, ${ }^{1}$ F. Bruni, ${ }^{1}$ M. A. Ricci, ${ }^{1}$ and A. K. Soper ${ }^{2,3}$ \\ ${ }^{1}$ Dipartimento di Fisica "E. Amaldi", Università degli Studi "Roma Tre”, Via della Vasca Navale 84, 00146 Roma, Italy \\ ${ }^{2}$ Isis Facility, Rutherford Appleton Laboratory, Chilton, Didcot, Oxfordshire, OX11 0QX, United Kingdom \\ ${ }^{3}$ Department of Physics and Astronomy, University College London, Gower Street, London WC1E 6BT, United Kingdom
}

(Received 22 October 2007; revised manuscript received 18 January 2008; published 28 August 2008)

\begin{abstract}
It is well established that at ambient and supercooled conditions water can be described as a percolating network of $\mathrm{H}$ bonds. This work is aimed at identifying, by neutron diffraction experiments combined with computer simulations, a percolation line in supercritical water, where the extension of the H-bond network is in question. It is found that in real supercritical water liquidlike states are observed at or above the percolation threshold, while below this threshold gaslike water forms small, sheetlike configurations. Inspection of the three-dimensional arrangement of water molecules suggests that crossing of this percolation line is accompanied by a change of symmetry in the first neighboring shell of molecules from trigonal below the line to tetrahedral above.
\end{abstract}

DOI: 10.1103/PhysRevE.78.021505

PACS number(s): 61.05.fm, 61.20.Ja, 61.25.-f

\section{INTRODUCTION}

In the late 1970s, Stanley [1] proposed the correlated-site polychromatic percolation model, as a useful tool for interpreting the anomalous properties of ambient and supercooled water. Since then this model has been corroborated by several tests on experimental data and computer simulations [2-4]. According to this model, liquid water can be considered as a "transient gel" or percolating H-bond network, which continuously reconstructs itself on the picosecond time scale of the $\mathrm{H}$ bonds. Recently the concept of percolation has been applied to analyze the structure of supercritical water $[5,6]$, in order to identify a percolation line, which separates two structurally different fluids, respectively below and above the so-called percolation threshold. This threshold is defined as the locus of thermodynamic states where the distribution of cluster size, $P(n)$, obeys the power law

$$
P(n) \sim n^{-\tau}
$$

with the universal exponent $\tau=2.19$ in three-dimensional systems $[7,8]$. In ambient and supercooled states water is a fully percolating system, since the average number of $\mathrm{H}$ bonds per molecule exceeds the critical value $n_{c}=1.55$ [4] in these conditions. The existence of low-density and lowconnectivity states instead below the percolation threshold has recently been predicted in the supercritical fluid by Pártay and co-workers $[5,6]$. As a matter of fact, the percolation theory seems a natural framework for describing the density fluctuations and clustering of molecules in supercritical fluids (including simple ones [9]), and the persistence of a small $\mathrm{H}$-bond peak in the radial distribution function of supercritical water [10-14] unambiguously defines a bound pair, based on a geometrical criterion. Thus water, even in supercritical states, is a paradigmatic sample for percolation studies $[15,16]$. We notice also that, although the concept of percolation implies in principle formation of an infinite cluster, which may only occur in an infinite system, Eq. (1) defines a percolation threshold also in a finite system, such as a simulation box.
So far studies of percolation phenomena have been used to qualitatively justify the behavior of experimental data and quantitative tests have been limited to model systems, through the analysis of the outcome of molecular dynamics or Monte Carlo simulations. Since the development of the empirical potential structure refinement (EPSR) [17-20] code for the analysis of neutron and x-ray diffraction data from liquid and amorphous materials, experimentalists also have access to a collection of molecular configurations. These can be investigated and represented in the same manner as for the output of a classical computer simulation, with the advantage of being compatible with an experiment performed on a real sample. Consequently a test of the H-bond percolation model based on experimental data is now feasible.

In this work we present the EPSR analysis of new and old [13] neutron diffraction data from supercritical water, in the framework of the percolation model, with the aim of investigating the structural differences between gaslike and liquidlike states and the possible location of the percolation line of real water. The crossing of this line determines important changes in the connectivity of the fluid, which may influence the properties of supercritical water as a solvent and oxidizing medium for organic and toxic waste [21].

\section{EXPERIMENTAL METHODS AND DATA ANALYSIS}

The thermodynamic states investigated have been chosen to be as close as experimentally feasible to the thermodynamic states investigated in Ref. [5] and labeled after that work, as shown in Table I. All experimental points are at pressures and temperatures above water critical values, namely, $P_{c}=220.64$ bar and $T_{C}=647.096 \mathrm{~K}$, while only the state point $A^{\text {expt }}$ corresponds to a density lower than the critical one $\left(\rho_{c}=332 \mathrm{~kg} / \mathrm{m}^{3}\right)$, as shown in Fig. 1. Neutron diffraction measurements were performed on the SANDALS [23] diffractometer, installed at the ISIS facility [24] (U.K.). The sample container was a Ti-Zr cell designed to withstand a pressure of $3 \mathrm{kbar}$ : it consists of five cylindrical holes $(1.7$ $\mathrm{mm}$ internal diameter) drilled in a slab $(0.75 \mathrm{~cm}$ thickness, 
TABLE I. Temperature, pressure, and density of the investigated states [22].

\begin{tabular}{lccl}
\hline \hline Label & $T(\mathrm{~K})$ & $P($ bar $)$ & $\rho\left(\mathrm{g} / \mathrm{cm}^{3}\right)$ \\
\hline$A^{\text {expt }}$ & 673 & 250 & 0.116 \\
$B^{\text {*expt }}$ & 673 & 500 & 0.58 \\
$B^{\text {expt }}$ & 673 & 1500 & 0.75 \\
$D^{\text {expt }}$ & 753 & 2450 & 0.75 \\
\hline
\end{tabular}

$4.05 \mathrm{~cm}$ lateral width) [25] and connected to a pressure rig. Temperature control was achieved by heaters in contact with the top and bottom of the sample container, giving a temperature stability better than $\pm 0.1 \mathrm{~K}$. Three samples, namely, pure $\mathrm{D}_{2} \mathrm{O}$, pure $\mathrm{H}_{2} \mathrm{O}$, and an equimolar mixture of the two were prepared at liquidlike densities, in order to exploit the isotopic substitution in the neutron diffraction experiment (NDIS) [26]. At state point $A^{\text {expt }}$ only the fully deuterated and the H-D equimolar mixture were measured, since the signal-to-noise ratio for the fully hydrogenated sample was prohibitive. The total acquisition time on each sample was between 8 and $10 \mathrm{~h}$, with ISIS running on average at $180 \mu \mathrm{A} / \mathrm{h}$; data were recorded every $3 \mathrm{~h}$, in order to check the entire apparatus stability. Data from an already published experiment [13] at $T=673 \mathrm{~K}$ and $\rho=0.58 \mathrm{~g} / \mathrm{cm}^{3}$ have also been reanalyzed and labeled as $B^{* \text { expt }}$.

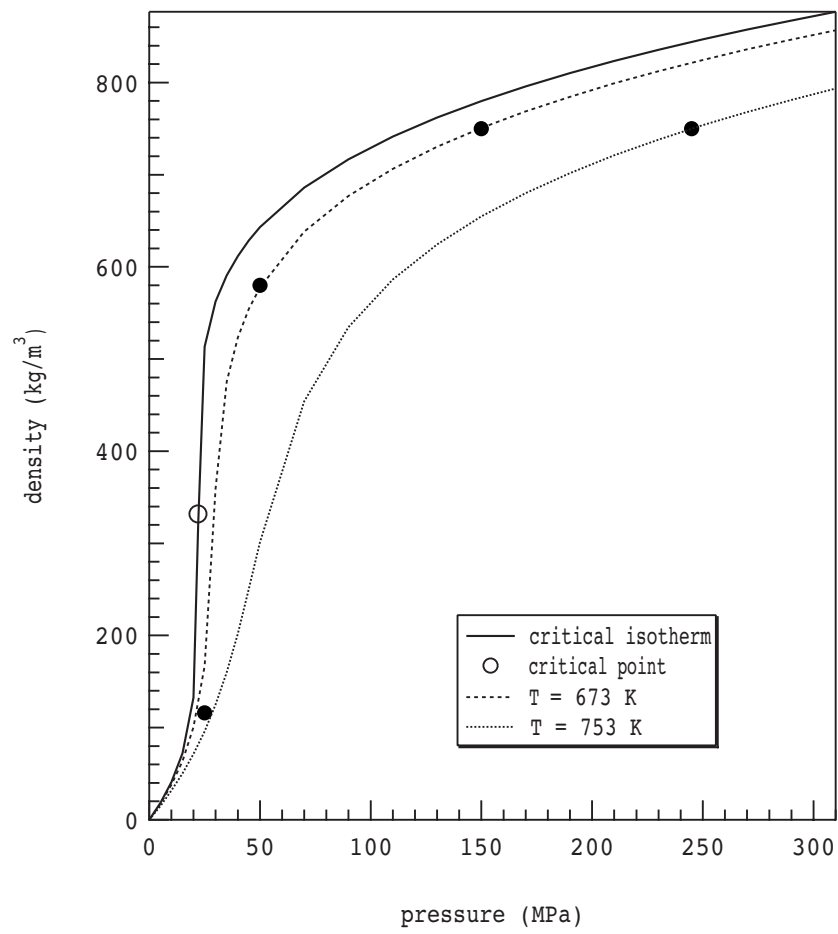

FIG. 1. Density and pressure values of the experimental points investigated are reported as solid circles; the empty circle represents the critical point of water. The critical isotherm $(T=647.096 \mathrm{~K}$, solid line) is also reported along with the isotherms at $T=673$ (dashed line) and $753 \mathrm{~K}$ (dotted line). All experimental temperatures and pressures exceed the critical values; the state at density lower than the critical one is in the so-called gaslike phase, all the others are in the liquidlike one.
Data reduction has been performed by using the GUDRUN routine available on SANDALS, which performs corrections for multiple scattering, absorption, and inelasticity effects, along with subtraction of the scattering from the sample container, and data reduction to an absolute scale, following the procedure described in the ATLAS manual [27]. The output of the GUDRUN routine is the interference differential cross section (IDCS), $F(Q)$, defined in Eqs. (2)-(4) and measured in b/at. sr:

$$
F(Q)=\Sigma_{\alpha} \Sigma_{\beta \geq \alpha} w_{\alpha \beta}\left[S_{\alpha \beta}(Q)-1\right],
$$

where $Q$, the momentum transferred in the interaction between a neutron and a nucleus, is defined as a function of the neutron wavelength $\lambda$ and scattering angle $2 \theta$ by

$$
Q=\frac{4 \pi}{\lambda} \sin \theta,
$$

and the weighting factors

$$
w_{\alpha \beta}=c_{\alpha} c_{\beta} b_{\alpha} b_{\beta}\left(2-\delta_{\alpha \beta}\right)
$$

depend on the neutron scattering lengths $b_{\alpha}$ and $b_{\beta}$ [28] of the $\alpha \beta$ atom pairs, while the Kronecker $\delta_{\alpha \beta}$ avoids double counting of like terms in the summation. The $S_{\alpha \beta}(Q)$ are called partial structure factors and are defined as Fourier transforms of the corresponding radial distribution functions (RDFs) of the $\alpha \beta$ pair, $g_{\alpha \beta}(r)$ :

$$
S_{\alpha \beta}(Q)-1=4 \pi \rho \int_{0}^{\infty} r^{2}\left[g_{\alpha \beta}(r)-1\right] \frac{\sin (Q r)}{Q r} d r .
$$

With the hypothesis that the microscopic structure of the deuterated sample does not sensibly differ from that of the hydrogenated one, the difference between the three IDCSs depends only on the different weighting factors and the maximum contrast among the experimental data is achieved for the H-D concentration used in the present experiment. In the case of pure water, three RDFs can be defined and extracted from the experimental data, namely, $g_{\mathrm{OO}}(r), g_{\mathrm{OH}}(r)$, and $g_{\mathrm{HH}}(r)$.

The experimental IDCSs have been used as input for the EPSR routine [17-20]. This is a Monte Carlo routine, which builds up a three-dimensional model of the sample that is constrained by the three independent experimental data sets. The routine requires as input the sample composition and thermodynamic parameters and a model for the site-site interactions, which will be used as "reference" or "seed" for the simulation. In the case of water, it is important that the interaction model contains suitable point charges in order to emulate the $\mathrm{H}$ bonding between molecules. There are several models in the literature and all of them give sensible results, in particular as far as the number of $\mathrm{H}$ bonds is concerned, when used within the EPSR code $[29,30]$. In the present case we have used the simple point charge/extended (SPC/E) [31] model as reference: this means that this model has been used to start the simulation and produce an initial equilibrated ensemble. We have used a cubic simulation box, containing 1000 molecules in liquidlike states and 250 molecules in state $A^{\text {expt }}$, with lateral dimension depending on the sample density. Periodic boundary conditions have been applied and 

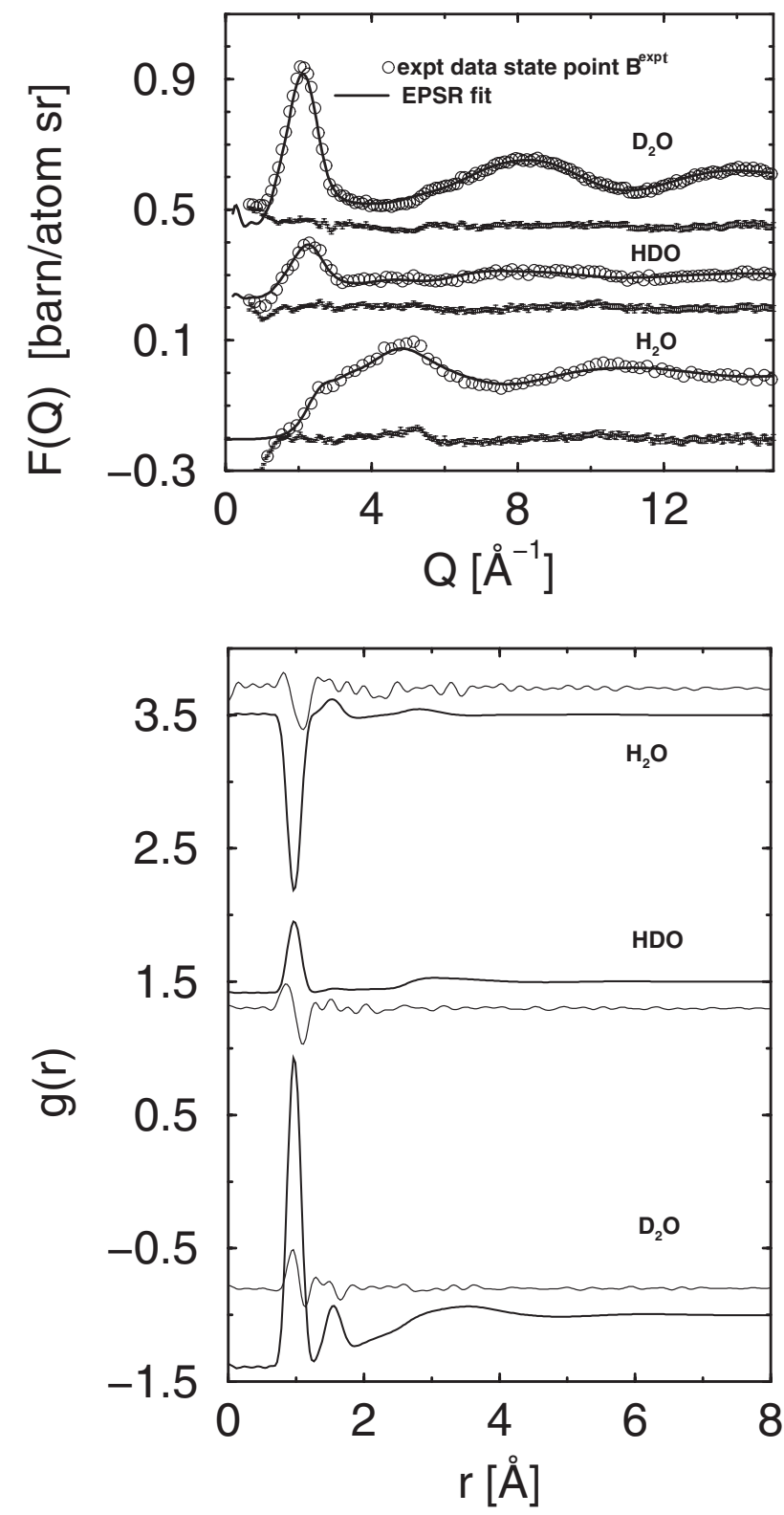

FIG. 2. Top: Experimental IDCSs (circles) along with their EPSR fit (solid line) for the three samples measured at state point $B^{\text {expt }}$. The fit residuals are reported with their error bars to show the substantial absence of residual structures. Data for $\mathrm{HDO}$ and $\mathrm{D}_{2} \mathrm{O}$ have been arbitrarily shifted for clarity. Bottom: Total neutron weighted radial distribution function for $\mathrm{H}_{2} \mathrm{O}, \mathrm{HDO}$, and $\mathrm{D}_{2} \mathrm{O}$ (thick solid line), compared with the Fourier transform of the datafit residuals (thin line). Data have been arbitrarily shifted for clarity.

the potential cutoff has been set to $12 \AA$. After equilibration, an empirical correction to the reference potential has been evaluated iteratively within the routine, in order to achieve the best fit to the experimental $F(Q)$ functions. This iterative procedure leads to the refinement of the so-called empirical potential, which will then be used while accumulating molecular configurations, during the production run. This enables the noise in the radial distribution functions to be reduced to an insignificant level, avoiding the direct Fourier transform of the experimental data, and giving access to

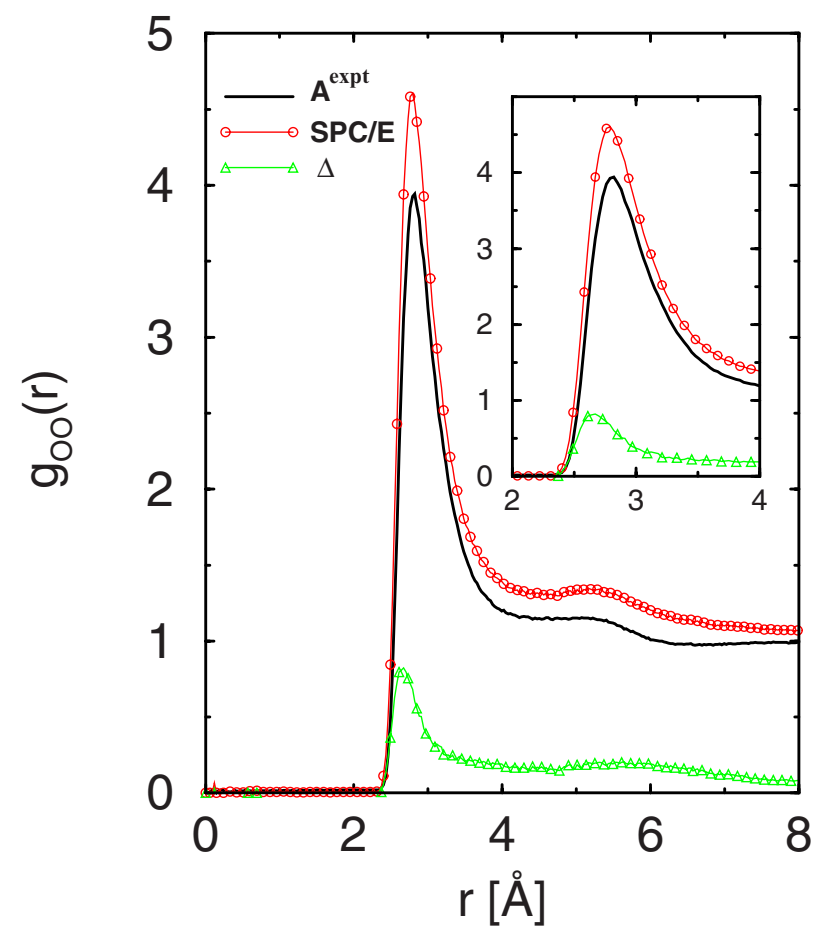

FIG. 3. (Color online) Comparison between the $g_{\mathrm{OO}}(r)$ function obtained at state point $A^{\text {expt }}$ by the EPSR routine after refinement of the empirical potential (black solid line) and by running with the $\mathrm{SPC} / \mathrm{E}$ reference potential alone (red line with circles). The green line with triangles is the difference of the two curves. The first neighbor peak is reported in the inset on a larger scale, in order to evidence the shift of the peak.

other structural quantities not otherwise available from the experiment.

The typical quality of an EPSR fit to the NDIS data is shown in Fig. 2, where the data relative to state point $B^{\text {expt }}$ are reported, as an example. The top panel shows the experimental data and their fit, along with the residual; the bottom panel shows the so-called total neutron weighted $g(r)$ functions (thick line), i.e., the Fourier transforms of the IDCS functions [Eq. (2)] for the three samples, along with the Fourier transform of the data-fit residuals (thin line). This figure demonstrates that for all three IDCS functions the residuals between data and fit are substantially due to differences in the intramolecular bond length between $\mathrm{D}_{2} \mathrm{O}$ and $\mathrm{H}_{2} \mathrm{O}$, while in the region of interest for this paper only the ripples due to truncation and experimental noise are visible. This issue has already been discussed in previous work [32,33]. Moreover, although differences between the empirical and the reference potentials are usually small and short ranged [18,34], nevertheless only the empirical potential allows reproduction of all features of the IDCS data within the EPSR simulation, with consequent recording of molecular configurations compatible with the experiment. In order to highlight this point, we have reported in Fig. 3 the oxygen-oxygen radial distribution function calculated by the EPSR code in the thermodynamic state $A^{\text {expt }}$ with and without switching on the empirical potential. The difference between these two site-site radial distribution functions is also plotted to underline the size and the range of the perturbation brought by the empirical potential. 


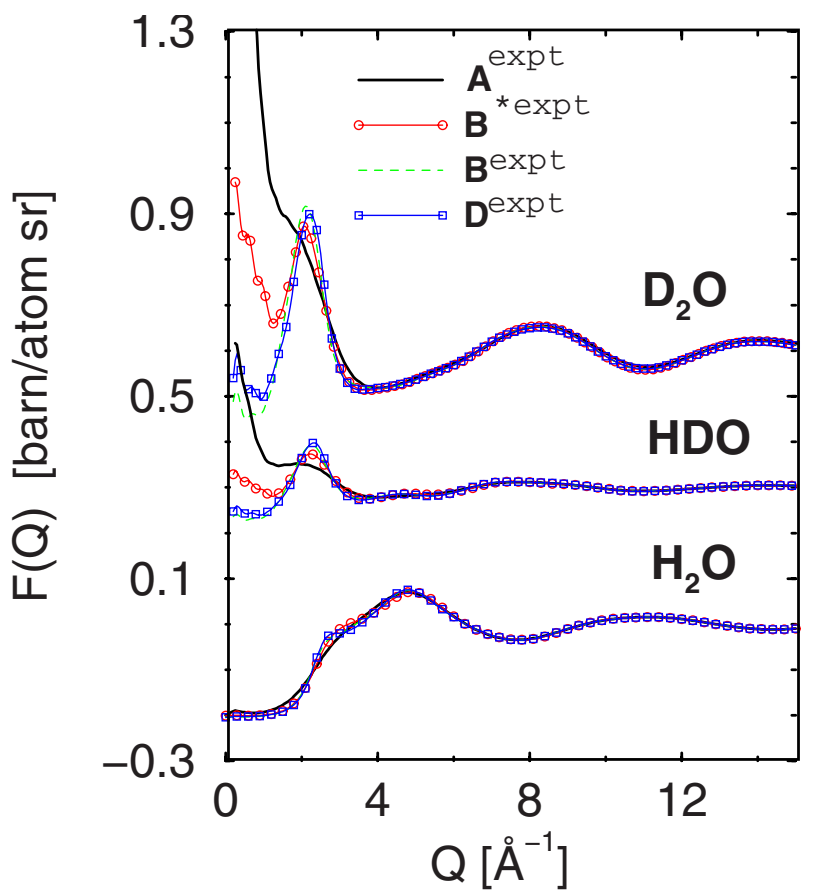

FIG. 4. (Color online) EPSR fits of the experimental data for the three isotopic compositions at all state point considered. Data for $\mathrm{HDO}$ and $\mathrm{D}_{2} \mathrm{O}$ have been arbitrarily shifted for clarity. Data in the gaslike region are reported in black; in red with circles the data from Ref. [13]; present data in the liquidlike region are reported in green (dashed line) $(T=673 \mathrm{~K})$ and blue with squares $(T$ $=753 \mathrm{~K}$ ). Changes of all functions for $Q$ values below $5 \AA^{-1}$ are evident, along with the increase of density fluctuations when the critical density is approached. These are visible in the $\mathrm{D}_{2} \mathrm{O}$ data and not in the $\mathrm{H}_{2} \mathrm{O}$, due to near cancellation between $b_{\mathrm{H}}$ and $b_{\mathrm{O}}$ $\left[\left(\bar{b}_{\mathrm{D}_{2} \mathrm{O}}\right)^{2}=0.407 \mathrm{~b} ;\left(\bar{b}_{\mathrm{H}_{2} \mathrm{O}}\right)^{2}=0.028\right]$.

The fits of the IDCS data, reported in Fig. 4, clearly show differences between the different state points. In particular in both $\mathrm{D}_{2} \mathrm{O}$ and HDO data the increase of density fluctuations for states $A^{\text {expt }}$ and $B^{* \text { expt }}$, as indicated by a pronounced low- $Q$ rise, in the vicinity of the critical point is evident, along with a shift of the first peak toward lower $Q$ values when the density or the temperature decreases. For $\mathrm{H}_{2} \mathrm{O}$ there is no equivalent rise because the mean scattering length for light water is close to zero, so these data are not sensitive to density fluctuations. These density fluctuations should be carefully distinguished from the cluster distributions to be discussed later. In the $\mathrm{H}_{2} \mathrm{O}$ data we notice instead that the low- $Q$ structure of the first peak gradually disappears as the temperature or the density decreases. These changes are mirrored in the site-site RDFs reported in Figs. 5-7. It has to be noted here that the uncertainty on the EPSR data (in both $Q$ and $r$ space) uniquely depends on the number of accumulated configurations (as for any simulation work), while the quality and signal-to-noise ratio of the experimental data are responsible for the reliability of the results. Moreover, the quantities of interest for the present study, and in particular the monotonic or oscillatory character of the $g(r)$ 's and the coordination or H-bond numbers are largely independent of the choice of reference potential [29,30].

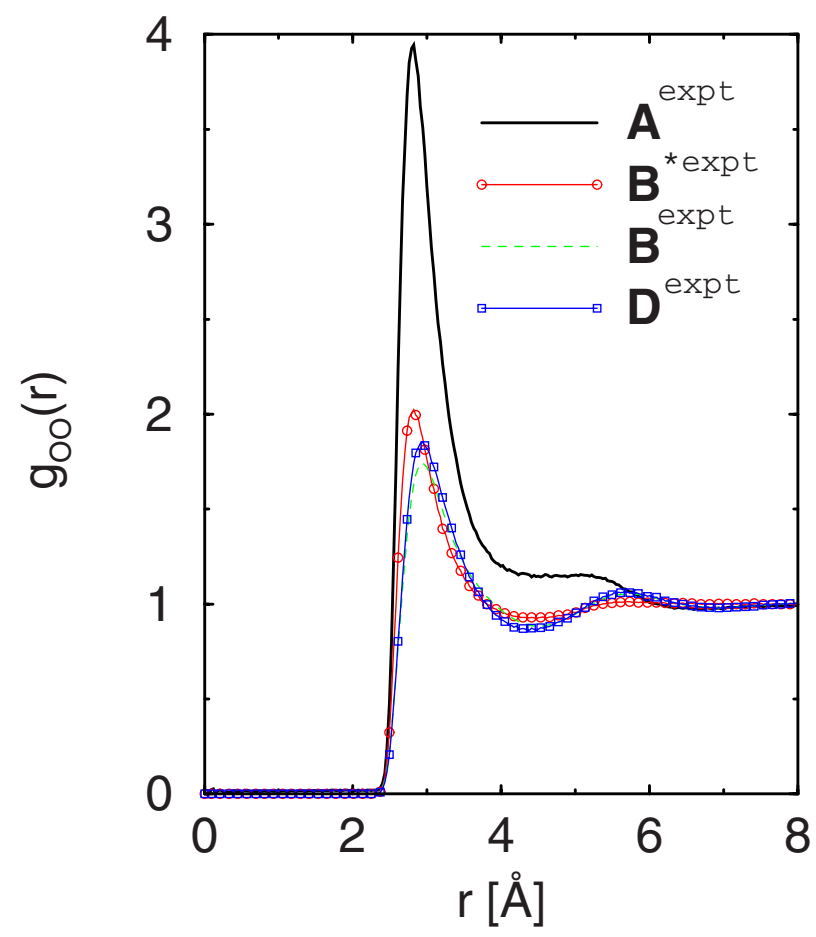

FIG. 5. (Color online) OO radial distribution functions at all investigated state points. Same symbols as for Fig. 4. Notice the gaslike behavior at state point $A^{\text {expt }}$ (see also Figs. 6 and 7).

\section{RESULTS AND DISCUSSION}

Inspection of the site-site radial distribution functions reported in Figs. 5-7 confirms that state point $A^{\text {expt }}$ falls in the gaslike region of the supercritical fluid, as its RDFs have very intense peaks at short distances and slowly decay to the $r \rightarrow \infty$ limit without oscillations. The RDFs obtained for the other three state points show instead liquidlike oscillations. In particular, the RDFs of states $B^{\text {expt }}$ and $D^{\text {expt }}$ are very close to each other and hardly distinguishable within the statistical accuracy of the simulation. More distinct differences are instead visible between states $B^{\text {expt }}$ and $D^{\text {expt }}$ and state $B^{* \text { expt }}$. In Fig. 5 we note that the first peak position of the $g_{\mathrm{OO}}(r)$ function at state point $B^{* \text { expt }}$ is shifted to shorter distances compared to the other two liquidlike states, while all three functions have the second neighbor shell centered in the range 5.60-5.85 $\AA$. Moreover both the H-bond peak in $g_{\mathrm{OH}}(r)$ and the first structure of the main peak of $g_{\mathrm{HH}}(r)$ are best resolved and visible at state point $B^{* \text { expt }}$. The shift of the second peak of the $g_{\mathrm{OO}}(r)$ function toward distances of the order of twice the first peak position is a well known feature of supercritical water and signals the breakdown of the tetrahedral H-bond network characteristic of ambient and supercooled water [13].

The fact that we observe oscillations in the RDFs for states $B^{\text {expt }}, B^{* \text { expt }}$, and $D^{\text {expt }}$, but not for state $A^{\text {expt }}$ is reminiscent of the Fisher-Widom (FW) behavior [35] observed in simpler systems. This line of state points separates the region where the RDF oscillates to larger $r$, corresponding to liquidlike behavior, from the region where it decays monotonically, corresponding to gaslike behavior. Hence it would appear from our data that state $A^{\text {expt }}$ lies on the gaslike side of 


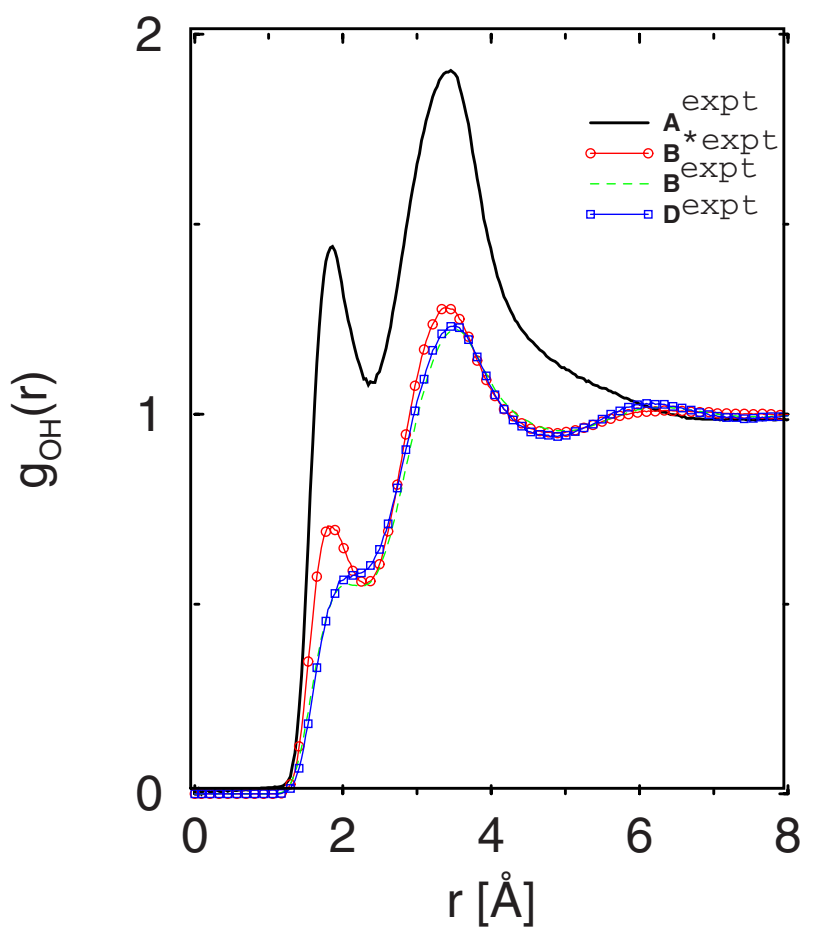

FIG. 6. (Color online) $\mathrm{OH}$ radial distribution functions at all investigated state points. Same symbols as in Fig. 4.

the FW line, while the other states are in the liquidlike region.

At state point $A^{\text {expt }}$ the number of first neighbor oxygen atoms per water molecule, obtained according to

$$
n\left(r^{*}\right)=\int_{0}^{r^{*}} 4 \pi \rho_{\mathrm{O}} r^{2} g_{\mathrm{OO}}(r) d r
$$

by integrating out to the position of the point of inflection after the main peak, i.e., $r^{*}=4.3 \AA$, is of the order of 1.8 , and on average each oxygen forms $\sim 0.42 \mathrm{H}$ bonds. After the critical density is crossed, both these numbers increase, and at liquidlike conditions the number of first neighbors becomes of the order of 8 and each oxygen forms on average 1 $\mathrm{H}$ bond. These numbers are to be compared with $\sim 4.5$ and $\sim 2$ at ambient conditions respectively, but note that in ambient water the position of the first minimum in $g_{\mathrm{OO}}(r)$ is at lower $r^{*}$, which is why the ambient coordination number is smaller than in the supercritical state.

The information discussed so far could also be achieved by standard Fourier transform of the experimental data, although well-known truncation artifacts and noisy data would have the effect of spoling the quality of the results in $r$ space. The availability of molecular configurations from the EPSR code has the advantage of eliminating systematic errors (as unfittable unphysical features) and at the same time allows investigation of the microscopic structure of the sample beyond the limits imposed by the traditional radial distribution functions, as in classical simulation work. In particular, we can analyze the orientational correlations between neighboring molecules through appropriate angular distributions and

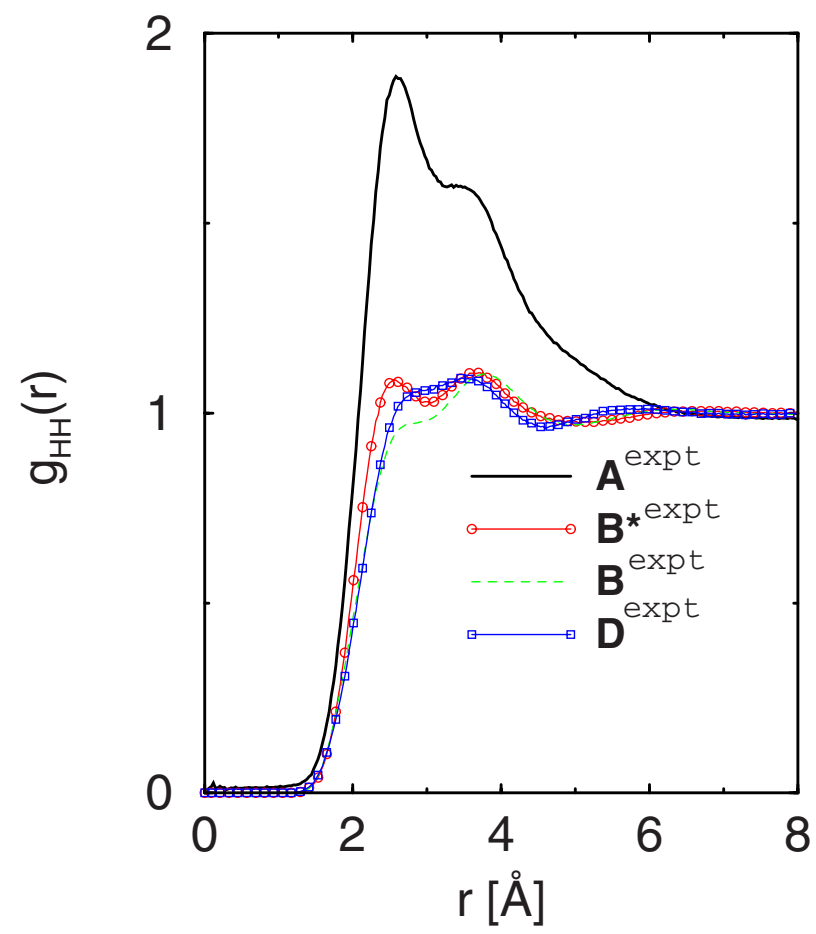

FIG. 7. (Color online) HH radial distribution functions at all investigated state points. Same symbols as in Fig. 4.

the spatial density of molecules in the neighborhood of a central one.

Figure 8 reports the distribution function of the angle $\theta$, formed between a molecule in the origin and two of its first neighbors, calculated accounting only for $\mathrm{OO}$ pairs within a distance of $3.5 \AA$ [i.e., the first minimum of the $g_{\mathrm{OO}}(r)$ function at ambient conditions]. This distribution at ambient conditions is characterized by an intense and broad peak centered near the tetrahedral angle $\left(109^{\circ}\right)$ and by a secondary peak at $\sim 55^{\circ}$, due to the presence of interstitial molecules. At supercritical conditions the peak at $104^{\circ}$ is hardly visible and possibly shifted at the highest densities (states $B^{\text {expt }}$ and $D^{\text {expt }}$ ) and totally disappears at state points $A^{\text {expt }}$ and $B^{* e x p t}$. The distribution functions are instead dominated by the lowangle peak, which becomes broader, going from state point $A^{\text {expt }}$ to state point $D^{\text {expt }}$. These angular correlations imply the absence of tetrahedral coordination with consequent reduction of the average number of $\mathrm{H}$ bonds per molecule and distortion of the intact bonds from linearity. The latter point is confirmed by the distribution function of the $\gamma$ angle, that is, the angle between the intramolecular $\mathrm{O}-\mathrm{H}$ bond and the $\mathrm{O}-\mathrm{O}$ director, reported in Fig. 9: this distribution becomes increasingly broader going from state point $A^{\text {expt }}$ to state point $D^{\text {expt }}$. We notice that also the secondary peak at $\sim 107^{\circ}$, corresponding to the angle formed by the O-O director with the second $\mathrm{O}-\mathrm{H}$ bond of the same molecule, is almost flat at supercritical conditions.

By developing the RDFs into their spherical harmonic components, it is possible to evaluate the spatial density functions (SDFs) of water, originally defined in Ref. [37]. These can be used for rendering in three dimensions (3D) the relative orientations of neighboring molecules, by identifying around a central molecule of given orientation the regions 


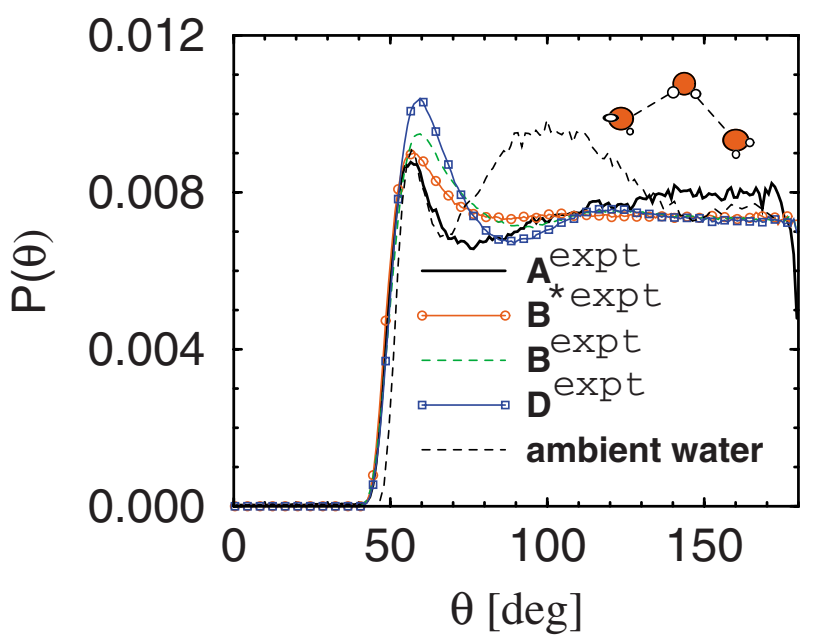

FIG. 8. (Color online) Distribution function of the angle $\theta$ formed between two oxygen atoms lying within a distance of $3.5 \AA$ from the oxygen in the origin of the reference frame. Same symbols as in Fig. 4 for supercritical water; the black dashed line refers to ambient water [36] and shows the peak characteristic of the tetrahedral coordination (at $104^{\circ}$ ), absent in supercitical states.

where the probability of finding another molecule, averaged over orientations, exceeds a threshold value. These functions have been plotted in Fig. 10 at all investigated thermodynamic states, for selected threshold (or contrast) values and maximum distances from the central molecule equal to the first and second minima of the $g_{\mathrm{OO}}(r)$ function. The first column shows that the first neighboring molecules of the central water are attracted toward the potential minima near the hydrogen sites or the lone pair electrons of the oxygens, in all investigated conditions. Interestingly, this function at state point $A^{\text {expt }}$ exhibits a triangular symmetry instead of the characteristic tetrahedral one. Moreover, as the probability threshold is lowered (contrast increased) the first neighbor shell is isotropically occupied in all investigated supercritical states (second column of Fig. 10) and an almost perfect spherical symmetry is recovered in the highest-density state

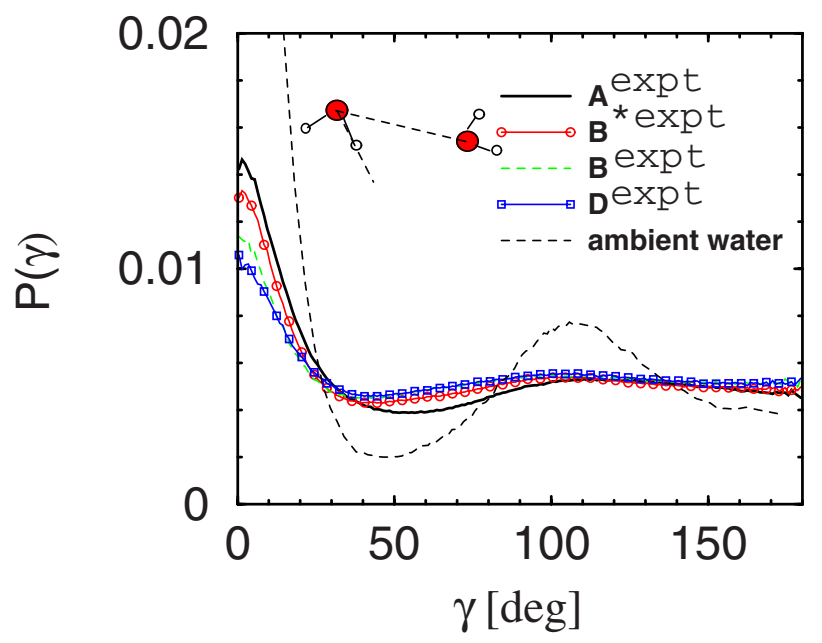

FIG. 9. (Color online) Distribution function of the H-bond angle $\gamma$. Same symbols as in Fig. 8. Note the significant weakening of the $\mathrm{H}$-bond linearity in supercritical water.

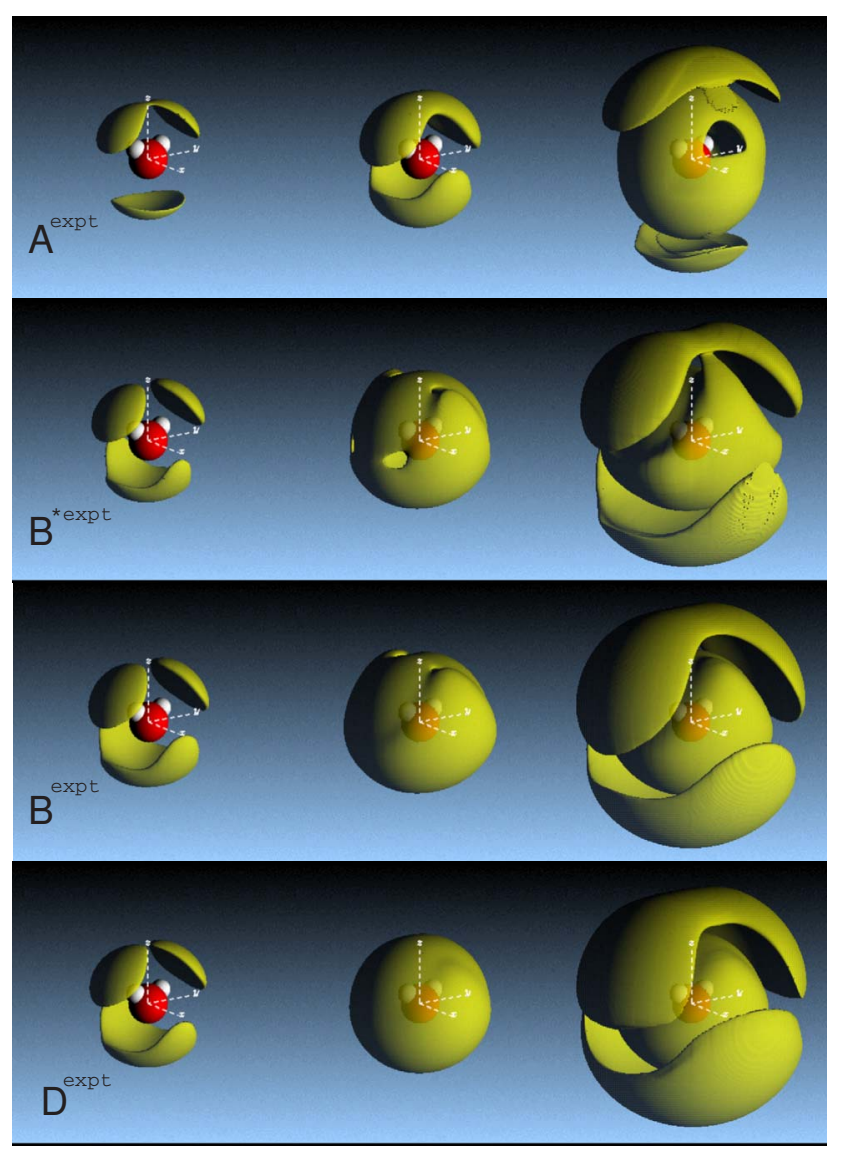

FIG. 10. (Color online) Spatial density functions for water molecules, however oriented, around a central molecule, giving a $3 \mathrm{D}$ rendering of the relative positions of neighboring molecules. The first row refers to state point $A^{\text {expt }}$, the second to state point $B^{\text {*expt }}$, the third to state point $B^{\text {expt }}$, and the fourth to state point $D^{\text {expt }}$. The first column shows the first neighboring shell with a contrast equal to 0.2 , while in the second the same $r$ range is reported with a contrast equal to 0.5 . The third column shows an $r$ range including both first and second neighboring shells, with a contrast equal to 0.4 . Notice the triangular symmetry of the SDFs at state point $A^{\text {expt }}$ in contrast with the characteristic tetrahedral one at the other state points for both first and second shells (first and last columns).

$\left(D^{\text {expt }}\right)$. The isotropic occupancy of the first neighbor shell is in agreement with the average breakdown of tetrahedral coordination. The third column of the figure reports the SDFs out to the second neighboring shell; it is interesting to note that, at all state points investigated, the second shell has the same shape as the first one, at variance with the SDFs of ambient water [37]. More importantly we stress that the trigonal symmetry of the SDF functions at state point $A^{\text {expt }}$ implies that water molecules are organized in small chains or sheets, while in the other states there are three-dimensional networklike clusters, although very small compared to those in ambient conditions.

In order to test the presence of percolating clusters of water molecules within our EPSR simulation boxes, for computational convenience we have adopted a geometrical definition of a H-bonded pair. Two water molecules are considered $\mathrm{H}$ bonded when the distance of a proton on one molecule from the oxygen of the second is less than or equal 


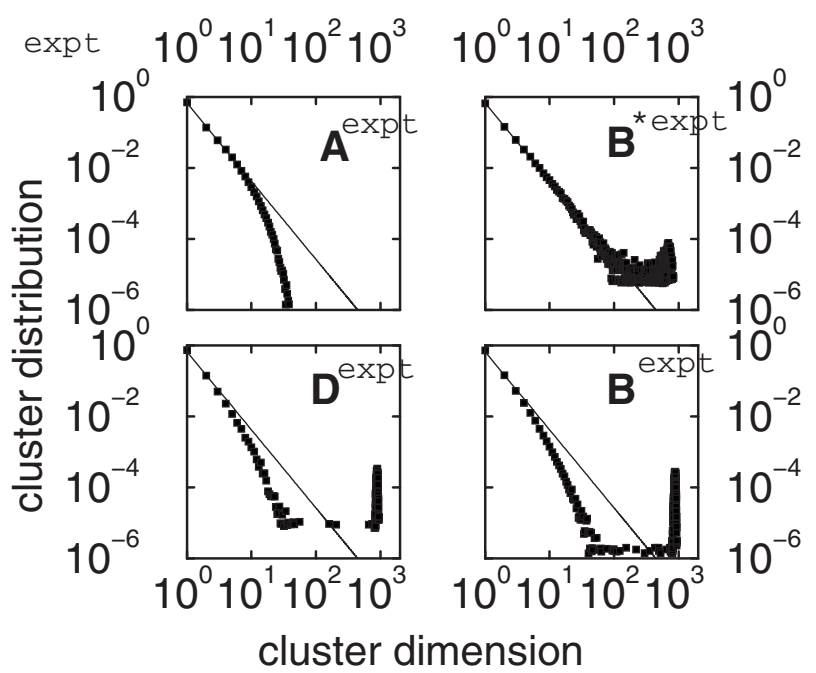

FIG. 11. Distribution function of clusters in the four investigated states. The theoretical distribution for a system at the percolation threshold [see Eq. (1)] is reported as the solid line, in order to highlight differences between percolating $\left(B^{* \text { expt }}, B^{\text {expt }}\right.$, and $\left.D^{\text {expt }}\right)$ and nonpercolating $\left(A^{\text {expt }}\right)$ states.

to the position of the first minimum of the $g_{\mathrm{OH}}(r)$ at ambient conditions (i.e., $2.4 \AA$ ). A group of molecules forms a cluster if each pair of molecules is bonded to at least one other molecule in the cluster. The distribution function of the clusters at all state points investigated is reported in Fig. 11, along with the threshold distribution $P(n)$ (solid line). At state points $B^{\text {expt }}$ and $D^{\text {expt }}$ the experimental distribution exceeds the threshold value at $n \sim 350$ and has a pronounced maximum at $n \sim 900$ molecules; finite-size effects are visible in the range $30 \leq n \leq 140$. State point $B^{* \operatorname{expt}}$ is at the percolation threshold in the entire range, while at state point $A^{\text {expt }}$ the distribution function falls below the threshold at $n$ $\geq 10$. These findings are in agreement with the simulations reported in Ref. [5] in similar thermodynamic states, and demonstrate that in supercritical water a percolation line can be defined as the locus of thermodynamic states which separates liquidlike from gaslike structures. In particular, state point $B^{* \text { expt }}$ seems to be very close to this line. Note that changes of the maximum distance of the $\mathrm{H}$ site on the second molecule by $\pm 0.1 \AA$ do not affect the qualitative results of our analysis, as shown in Fig. 12. Here comparison of the distribution functions of clusters at state points $A^{\text {expt }}$ and $B^{\text {expt }}$, calculated by using three different values of the maximum H-bond length, are shown. The distinction between percolating and nonpercolating states is thus independent of reasonable changes of the hydrogen-bond definition.

These findings are confirmed by the distribution functions of the fraction $F\left(i_{\mathrm{H}}\right.$ bond $)$ of water molecules engaged in $i \mathrm{H}$ bonds, reported in Fig. 13. The average number of $\mathrm{H}$ bonds per molecule at state point $A^{\text {expt }}, 0.8$, is below the percolation threshold value of 1.55 , and above this threshold for all the other thermodynamic state points. In particular, we find 2.2 bonds per molecule at state points $B^{\text {expt }}$ and $D^{\text {expt }}$ and 1.9 at state point $B^{* \text { expt }}$. In this state the frequency of molecules with only one bond is higher than that of molecules with three bonds, in contrast to what is found in the two highest-

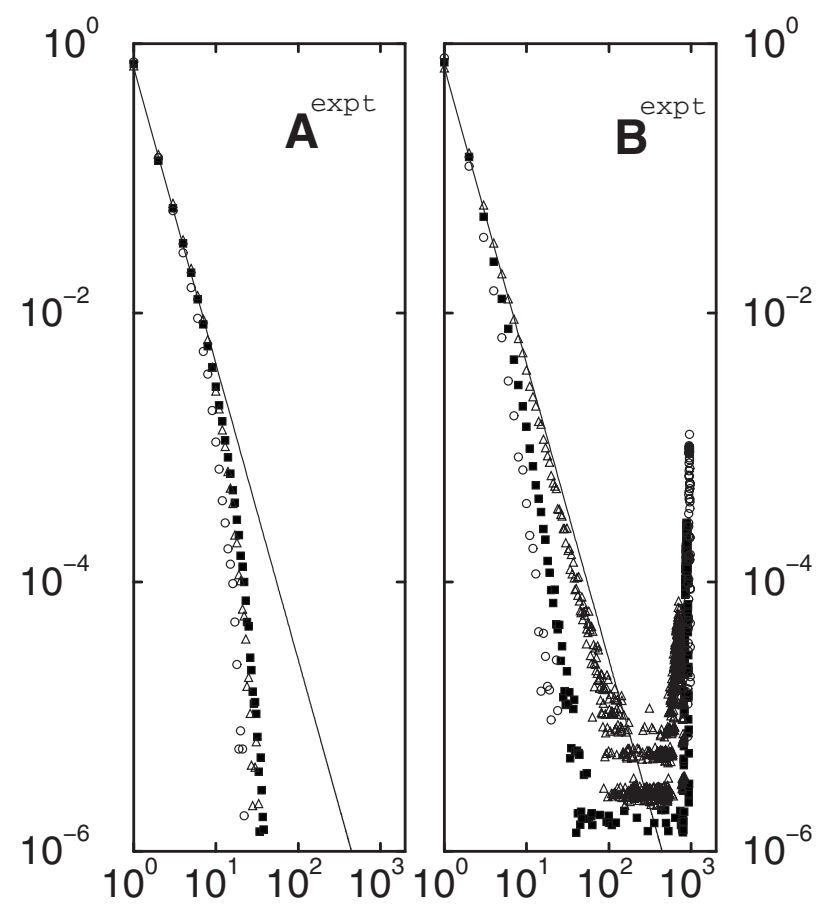

FIG. 12. In order to demonstrate that our conclusions about the presence of percolating water clusters in supercritical water are independent of the chosen maximum length of the $\mathrm{H}$ bond, we report here the distribution function of clusters at state points $A^{\text {expt }}$ and $B^{\text {expt }}$, calculated for different values of this length, namely, 2.3 (open circles), 2.4 (solid squares), and $2.5 \AA$ (open triangles). The theoretical distribution for a system at the percolation threshold [see Eq. (1)] is reported as solid line.

density states. It should be noted that we are referring here to the number of bonds per water molecule, that is, twice the number of acceptor bonds per oxygen atom. Assuming that $F\left(i_{\mathrm{H}}\right.$ bond $)$ is binomial, in each individual state we can evaluate the probability $p_{B}=F(4)^{0.25}$ that a $\mathrm{H}$ bond is intact; this simple calculation gives $p_{B}=0.214$ at state point $A^{\text {expt }}$ and $p_{B}=0.488,0.555$, and 0.567 in the other states, respectively, consistent with the value of $\sim 0.4$ at the percolation threshold [2-4].

Although in liquidlike states supercritical water is still a percolating system or very close to the percolation threshold, nevertheless the clusters do not reproduce a full 3D tetrahedral coordination, according to the $g(r)$ and distribution functions reported so far. In order to better investigate this point, we report in Fig. 14 the distribution functions of chains of molecules as a function of their length. This has been determined according to the condition that the first and last molecules of the chain are bonded only to one neighboring molecule and applying the shortest-path criterion for the measure of the chain length [38]. Also in this figure we note a clear difference between state point $A^{\text {expt }}$ and the other thermodynamic states. Indeed, in gaslike conditions the distribution function is dominated by monomers and small oligomers, while at higher densities the formation of longer chains is evident. The distribution is peaked at a chain length of $\sim 13$ water molecules in all liquidlike states, although being flatter at state point $B^{* \text { expt }}$, which is likely the closest to the percolation line. Moreover, in liquidlike states the chains are pos- 


\section{5}

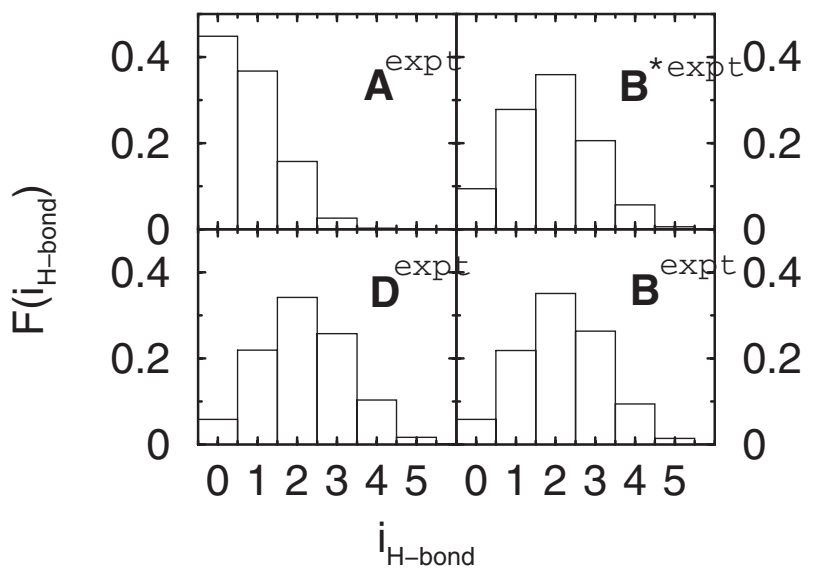

FIG. 13. Fraction of water molecules with $i \mathrm{H}$ bonds in the four states investigated. This distribution is almost symmetric and centered above the threshold value of $1.55 \mathrm{H}$ bonds per molecule at all liquidlike state points; at the gaslike state it is instead asymmetric and gives an average value of $0.8 \mathrm{H}$ bonds per molecule.

sibly branched to form three-dimensional clusters.

To summarize, it would appear, on the basis of these results, that there is a clear distinction in supercritical water between gaslike and liquidlike phases. The two states are separated by a percolation transition, in which water transforms from liquidlike behavior with three-dimensional percolating H-bond networks to gaslike short chains or sheets with local planar geometries, which do not percolate. More speculatively, it is notable that through this transition the radial distributions appear to transform from oscillatory to nonoscillatory, suggesting that the percolation line in a H-bonded system corresponds to the Fisher-Widom line in simpler fluids $[35,39]$. The present study is over too short a range to confirm this point definitively, but this topic certainly deserves further investigation.

\section{CONCLUSIONS}

By analyzing neutron diffraction data through the EPSR code, we have shown that a percolation line separates two structurally different aqueous fluids as the density decreases from values typical of a so-called liquidlike fluid to those of a gaslike system. It appears indeed that water even above its supercritical temperature can be considered as a highly dynamical percolating system, provided that the density is high enough (namely, $\rho \geq 1.8 \rho_{c}$ at $T=1.04 T_{c}$ ) for the radial distribution functions to show the oscillatory character typical of liquidlike systems. At low densities, when the radial distribution functions show gaslike behavior, water does not percolate. The transition is marked by a percolation line. Inter-

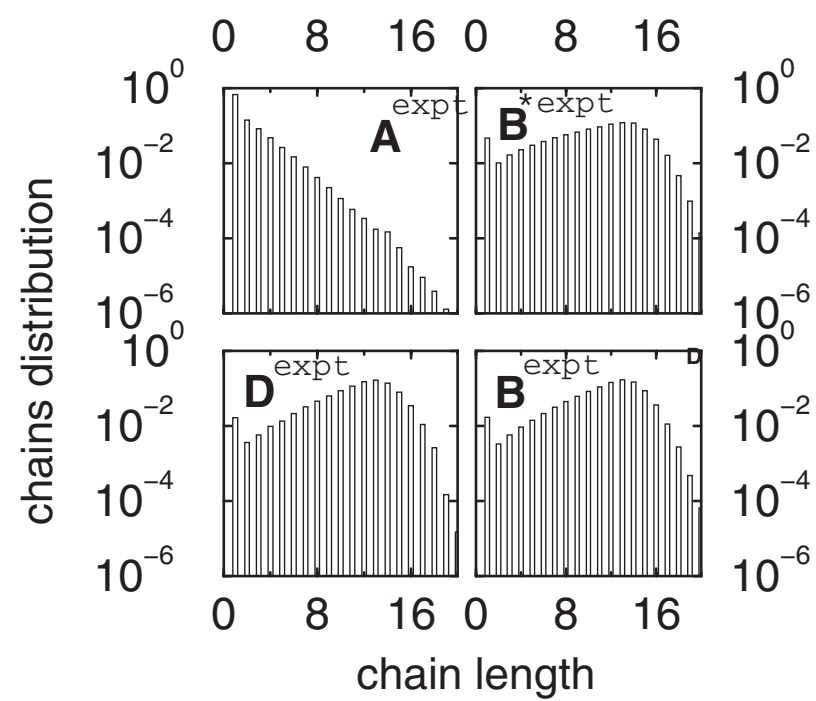

FIG. 14. Distribution function of chains of water molecules at state point $A^{\text {expt }}$ showing monotonic decrease with increasing chain length and a second maximum in liquidlike states.

estingly, the observation that this line separates gaslike from liquidlike states may also suggest a possible relation with the Fisher-Widom line [35,39], defined for simple liquids.

More importantly, the transition through the percolation line is accompanied by clear changes in the threedimensional structure of the fluid. In particular, the preferred tetrahedral arrangement of first neighboring molecules persists in liquidlike densities, while a triangular symmetry is found in gaslike states. This is correlated with the average number of $\mathrm{H}$ bonds per molecule, which decreases from $\sim 2$ to $\sim 0.8$ upon the transition; in other words, when the probability of finding a four-bonded molecule is below the threshold value of $\sim 0.25 \%$ (as for instance at state point $A^{\text {expt }}$, the tetrahedral spatial arrangement becomes unlikely. As a matter of fact the distribution functions reported in Fig. 13 predict that within an ensemble of 1000 water molecules only two form four $\mathrm{H}$ bonds at state point $A^{\text {expt }}$, while this number becomes of the order of 50 or 100 in the liquidlike states. Consequently, while liquidlike supercritical water can be still described as a percolating system formed by threedimensional clusters or branched chains, at gaslike densities water molecules are predominantly isolated or organized in small sheetlike oligomers.

\section{ACKNOWLEDGMENTS}

The authors wish to thank P. Jedlovszky for useful discussions. This work has been performed within the CCLRCCNR Agreement No. 01/9001, concerning collaboration in scientific research at the spallation neutron source ISIS and with partial financial support of CNR. 
[1] H. E. Stanley, J. Phys. A 12, L329 (1979).

[2] H. E. Stanley and J. Teixeira, J. Chem. Phys. 73, 3404 (1980).

[3] A. Geiger and H. E. Stanley, Phys. Rev. Lett. 49, 1895 (1982).

[4] R. L. Blumberg, H. E. Stanley, A. Geiger, and P. Mausbach, J. Chem. Phys. 80, 5230 (1984).

[5] L. Pártay and P. Jedlovszky, J. Chem. Phys. 123, 024502 (2005).

[6] L. Pártay, P. Jedlovszky, I. Brovchenko, and A. Oleinikova, Phys. Chem. Chem. Phys. 9, 1341 (2007).

[7] D. Stauffer, Introduction to Percolation Theory (Taylor \& Francis, London, 1985).

[8] A. Oleinikova, I. Brovchenko, A. Geiger, and B. Guillot, J. Chem. Phys. 117, 3296 (2002).

[9] N. Yoshii and S. Okazaki, J. Chem. Phys. 107, 2020 (1997).

[10] A. K. Soper, F. Bruni, and M. A. Ricci, J. Chem. Phys. 106, 247 (1997).

[11] M. C. Bellissent-Funel, T. Tassaing, H. Zhao, D. Beysens, B. Guillot, and Y. Guissani, J. Chem. Phys. 107, 2942 (1997).

[12] P. Jedlovszky, J. P. Brodholt, F. Bruni, M. A. Ricci, A. K. Soper, and R. Vallauri, J. Chem. Phys. 108, 8528 (1998).

[13] A. Botti, F. Bruni, M. A. Ricci, and A. K. Soper, J. Chem. Phys. 109, 3180 (1998).

[14] T. Tassaing, M. C. Bellissent-Funel, B. Guillot, and Y. Guissani, Europhys. Lett. 42, 265 (1998).

[15] R. Mountain, J. Chem. Phys. 110, 2109 (1999).

[16] A. G. Kalinichev and S. V. Churakov, Fluid Phase Equilib. 183, 271 (2001).

[17] A. K. Soper, Chem. Phys. 202, 295 (1996).

[18] A. K. Soper, Chem. Phys. 258, 121 (2000).

[19] A. K. Soper, Mol. Phys. 99, 1503 (2001).

[20] D. T. Bowron, J. L. Finney, and A. K. Soper, J. Phys. Chem. B 102, 3551 (1998).

[21] M. Akiya and P. E. Savage, Chem. Rev. 102, 2725 (2002).
[22] NIST Chemistry Webbook, www.webbook.nist.gov

[23] A. K. Soper, in Proceedings of the Conference on Advanced Neutron Sources 1988, edited by D. K. Hyer, IOP Conf. Proc. No. 97 (Institute of Physics and Physical Society, London, 1989), pp. 353-366.

[24] www.isis.rl.ac.uk

[25] www.isis.rl.ac.uk/disordered

[26] A. K. Soper, in Methods in the Determination of Partial Structure Factors, edited by J. B. Suck, D. Raoux, P. Chieux, and K. C. Riekel (World Scientific, Singapore, 1993), p. 58-63; A. K. Soper and J. Z. Turner, Int. J. Mod. Phys. B 7, 3049 (1993).

[27] www.isis.rl.ac.uk/disordered/Sandals/ATLASmanualDTB.pdf

[28] V. F. Sears, Neutron News 3, 26 (1992).

[29] F. Bruni, M. A. Ricci, and A. K. Soper, in Francesco Paolo Ricci: His Legacy and Future Perspectives of Neutron Spectroscopy, edited by M. Nardone and M. A. Ricci (Italian Physical Society, Bologna, 2000), p. 37-48.

[30] M. Bernabei, Laurea Thesis, Universitá di Roma Tre, Italy.

[31] H. J. C. Berendsen, J. R. Grigera, and T. P. Straatsma, J. Phys. Chem. 91, 6269 (1987)

[32] A. K. Soper, and C. J. Benmore (unpublished).

[33] A. Botti, F. Bruni, R. Mancinelli, M. A. Ricci, F. Lo Celso, R. Triolo, F. Ferrante, and A. K. Soper, J. Chem. Phys. 128, 164504 (2008).

[34] R. Mancinelli, A. Botti, F. Bruni, M. A. Ricci, and A. K. Soper,J. Phys. Chem. B 111, 13570 (2007).

[35] M. E. Fisher and B. Widom, J. Chem. Phys. 50, 3756 (1969).

[36] Data from Ref. [18] have been reanalyzed.

[37] I. M. Svishchev and P. G. Kusalik, J. Chem. Phys. 99, 3049 (1993).

[38] D. S. Franzblau, Phys. Rev. B 44, 4925 (1991).

[39] R. J. F. Leote de Carvalho, R. Evans, D. C. Hoyle, and J. R. Henderson, J. Phys.: Condens. Matter 6, 9275 (1994). 